\section{Case Reports in Ophthalmology}

\title{
Vitrectomy for Tractional Retinal Detachment with Twin Retinal Capillary Hemangiomas in a Patient with Von Hippel-Lindau Disease: A Case Report
}

\author{
Hiroyuki Suzuki ${ }^{a} \quad K^{2}$ igo Kakurai, b Seita Morishita ${ }^{a}$ Daisaku Kimura ${ }^{a, c}$ \\ Masanori Fukumoto $^{a}$ Takaki Sato ${ }^{a, c}$ Teruyo Kida ${ }^{a}$ Mari Ueki ${ }^{a}$ \\ Jun Sugasawa ${ }^{a}$ Tsunehiko Ikeda ${ }^{a}$ \\ ${ }^{a}$ Department of Ophthalmology, Osaka Medical College, Takatsuki City, Japan; \\ ${ }^{b}$ Department of Ophthalmology, Yao Tokushukai Hospital, Yao City, Japan; \\ 'Department of Ophthalmology, Takatsuki Red Cross Hospital, Takatsuki City, Japan
}

\section{Keywords}

Von Hippel-Lindau disease - Retinal capillary hemangiomas - Tractional retinal detachment . Laser photocoagulation · Vitrectomy

\begin{abstract}
Purpose: The purpose of this study was to report a case of Von Hippel-Lindau disease (VHL) with twin retinal capillary hemangiomas that was successfully treated by vitreous surgery for tractional retinal detachment following laser photocoagulation. Case: A 44-year-old male presented at our university hospital after noticing decreased visual acuity in his right eye. The patient had previously undergone multiple operations for cerebellar, thoracic, and lumbar spine hemangioblastomas when he was approximately 19 years old. Upon initial examination, ocular findings revealed twin connected retinal capillary hemangiomas around the temporal upper area of the patient's right eye. The patient was subsequently diagnosed with VHL based on his medical history and current observations of the ocular fundus. Tractional retinal detachment had occurred as the result of the formation of proliferative membranes following
\end{abstract}


Case Reports in
Ophthalmology

Case Rep Ophthalmol 2016;7:332-339 $10.1159 / 000446638$

(C) 2016 The Author(s). Published by S. Karger AG, Basel www.karger.com/cop

Suzuki et al.: Vitrectomy for Tractional Retinal Detachment with Twin Retinal Capillary Hemangiomas in a Patient with Von Hippel-Lindau Disease: A Case Report

laser photocoagulation. The patient underwent vitreous surgery to treat the tractional retinal detachment, resulting in a successful postoperative outcome. Conclusion: The findings of this study show the possibility that proliferative changes and tractional retinal detachment can arise following photocoagulation for retinal capillary hemangiomas in patients with VHL.

(c) 2016 The Author(s)

Published by S. Karger AG, Basel

\section{Introduction}

Von Hippel disease, an abnormality of a tumor-suppressing gene at the tip of the p-arm of chromosome 3, is a condition which reportedly takes the form of autosomal dominant inheritance as its mode of inheritance [1]. In cases where congenital retinal capillary hemangiomas are found in observations of the eye associated with the presence of tumors in the central nervous system, the condition is called 'Von Hippel-Lindau disease' (VHL). Reportedly, the peripheral area of the retina accounts for the site of occurrence in approximately $90 \%$ of retinal capillary hemangioma cases, whereas the optic papilla or peripapillary retina account for the site of occurrence in approximately $10 \%$ of the cases [2]. Although laser photocoagulation is the typical treatment for retinal capillary hemangiomas in the peripheral areas for Von Hippel disease, vitrectomies may be applied for cases in which tractional retinal detachment progresses [3-7]. In this present study, we report on a case in which vitrectomy was performed on a patient with VHL with twin connected retinal capillary hemangiomas who experienced tractional retinal detachment following laser photocoagulation.

\section{Case Report}

\section{Patient}

We report on a 44-year-old male with the chief complaint of decreased visual acuity (VA) in the right eye. The patient had previously undergone multiple operations for cerebellar, thoracic, and lumbar spine hemangioblastomas when he was approximately 19 years old. A laser photocoagulation had been performed on the patient at another hospital for a retinal capillary hemangioma in the right eye. Family history revealed that the patient's younger sister had blindness in her right eye due to VHL.

\section{History of Present Illness}

In September 2010, the patient became aware of myodesopsia in his right eye with a gradual decrease of vision, thus prompting him to seek examination and treatment by a nearby physician. Upon examination, he was diagnosed as having a retinal tumor in the right eye, and was subsequently referred to our department on October 22, 2010 for further treatment.

\section{Initial Observations}

Upon initial examination, the patient's VA was $0.02(0.05 \times$ S-5.25D) OD and $0.09(1.0 \times$ S-4.25D) OS, and hisiIntraocular pressure was $17 \mathrm{~mm} \mathrm{Hg}$ OD and $16 \mathrm{~mm} \mathrm{Hg}$ OS. Nothing noteworthy was observed in the anterior segment of either eye, and observations of the ocular fundus showed no abnormalities in the left eye. However, around the temporal upper area of the right eye, twin connected retinal capillary hemangiomas were found with papillary diameter sizes of 2 and 3.5, and peripheral fibrin deposition and exudative retinal de- 


\section{Case Reports in Ophthalmology}

tachment extending to the posterior pole of the eye were found (fig. 1a). Scarring formations presumed to be caused by the laser photocoagulation were also found around the hemangioma on the peripheral side.

\section{Clinical Course}

The patient was diagnosed with VHL based on his medical history and current observations of the ocular fundus. The exudative retinal detachment had reached into the second quadrant, including the posterior pole, and the patient was subsequently administered a sub-Tenon's capsule triamcinolone acetonide (STTA) $(20 \mathrm{mg} / 0.5 \mathrm{ml})$ injection on November 5,2010 to reduce inflammation. One month later, there was a marked decrease in subretinal fluid and VA had improved to 0.25 . Fluorescein angiography (FA) performed on January 20, 2011 revealed substantial leakage from the hemangioma on the peripheral side (fig. 1b), and laser photocoagulation was subsequently performed for treatment. The setting irradiating conditions were $500 \mu \mathrm{m}, 300 \mathrm{~ms}$, and $300-500 \mathrm{~mW}$, performed in 12 shots until the hemangioma became whitish in color. At a follow-up examination on February 4, 2011 an exudative retinal detachment and an increase in fibrin membranes were discovered, and his VA had decreased to 0.1 . Thus, an STTA $(20 \mathrm{mg} / 0.5 \mathrm{ml})$ was once again administered. A follow-up examination on May 6th, 2011 revealed the occurrence of tractional retinal detachment due to formation of proliferative membranes connected to the hemangioma, and that VA had decreased to 0.03 (fig. 2a). On May 31, 2011, the patient underwent pars plana vitrectomy, pars plana lensectomy, an encircling procedure, and a gas tamponade. During the operation, the peripheral hemangioma was extracted using vitreous scissors, and an endolaser photocoagulation ( $300 \mathrm{~mW}, 1,000 \mathrm{~s}, 17$ shots) was applied to the 3.5 papilla diameter hemangioma on the posterior side. As a thickened posterior vitreous membrane was found in the area of the hemangioma's posterior tractional retinal detachment, an artificial posterior vitreous detachment was successfully created. The tractional retinal detachment recovered following the operation, and at a follow-up examination in October 2011, no reproliferation was found, the hemangioma was found to have scarred slightly (fig. 2b), and the patient's VA had improved to 0.6 .

\section{Pathological Examination}

During a pathological examination of the tissue of the extracted hemangioma, an aggregation of blood vessels was confirmed in part of the hyalinized tissue via hematoxylin-eosin staining (fig. 3).

\section{Discussion}

VHL is a congenital condition which gives rise to malignant tumors in multiple organs, such as hemangioblastomas in the brain and spinal cord, as well as renal cancer and retinal capillary hemangiomas [1]. The condition presents with an autosomal dominant inheritance [2]. Ophthalmologically, occurrence of hemangiomas in the area surrounding the retina is common, though occasionally hemangiomas occur in the vicinity of the papilla, causing vitreous hemorrhage, macular pucker, and exudative or tractional retinal detachment if the condition progresses. In terms of the treatment for retinal capillary hemangiomas, laser coagulation is generally the first choice for peripheral hemangiomas within 2 papilla diameters in size [8]; however, and even though it is often difficult to treat sizeable hemangiomas and hemangiomas in the vicinity of the papilla, surgeries such as transscleral cryotherapy [9], 


\section{Case Reports in Ophthalmology}

transpupillary thermotherapy [10], photodynamic therapy [11], and anti-VEGF therapy [12] have reportedly all been used. Furthermore, in the event that vitreous hemorrhage, macular pucker, tractional retinal detachment, or similar reactions occur in resistance to these treatments, vitreous surgery is usually indicated [3-7]. Although direct intraocular lasers and diathermy are applied to the hemangioma during the operation, it has been reported that recurrences can arise and that the tumors can be removed with the objective of a complete cure [5]. Other reports claim that scleral buckling procedures and relaxing retinotomies were used in combination in cases of tractional retinal detachment in which retinal extension cannot be achieved solely through vitreous resection $[9,13]$. In cases where the tumor is extremely large, a method involving extraction of the tumor along the entire scleral wall of the eye has been reported [14].

In the present case, the patient underwent laser photocoagulation 25 years prior, and it is conceivable that this case was a recurrence of symptoms that had temporarily subsided. As no laser scarring was observed in the hemangioma on the posterior side, it is also conceivable that this was a new hemangioma that developed sometime after the previous treatment. With respect to the twin connected hemangiomas, laser photocoagulation was performed on the hemangioma on the peripheral side, however, proliferative changes arose following the photocoagulation and tractional retinal detachment occurred, thus leading the patient to undergo a vitrectomy. We theorize that the laser photocoagulation might have induced the intraocular inflammation which caused the vitreoretinal membrane formation.

During the operation, the hemangioma on the peripheral side was extracted, while the posterior hemangioma was treated with endolaser photocoagulation upon considering the complications of resection. We determined that the retinal hemangiomas of the posterior pole had a small bonding area with the retina, so even if it was extracted, invasion into the retina would be a small one. However, the retinal hemangiomas of the peripheral portion had a large bonding area with the retina and many vascular components within the tumor, so we determined that its extraction had the risk of causing massive bleeding. A relaxing retinotomy around the hemangioma was not indicated in this case because the thickened posterior vitreous membrane removed from the retina during the operation seemed to be the primary cause of the tractional retinal detachment. The reason for performing the encircling procedure was to loosen the vitreoretinal traction which was caused by the postoperative shrinkage of residual vitreous gel, and the reason for simultaneously performing gas tamponade at the same time was to reposition the tractional retinal detachment at an earlier stage.

The retina was successfully reattached following the operation and the posterior hemangioma was scarred, although the vascular composition inside the tumor persists and, despite the narrowing of the inflowing vessels, has been found to remain the same. It should be noted that McDonald et al. [4] reported disease recurrence in 3 of 10 cases that required vitrectomy, so the possibility remains that the present case could possibly experience disease recurrence in the future. Thus, it is important to perform strict and careful follow-up examinations.

\section{Acknowledgement}

The authors wish to thank John Bush for editing the manuscript. 


\section{Statement of Ethics}

This case study has been approved by the Ethics Committee of the Osaka Medical College.

\section{Disclosure Statement}

The authors have no conflicts of interest to disclose.

\section{References}

1 Haddad NM, Cavallerano JD, Silva PS: Von Hippel-Lindau disease: a genetic and clinical review. Semin Ophthalmol 2013;28:377-386.

2 Maher ER, Neumann HP, Richard S: von Hippel-Lindau disease: a clinical and scientific review. Eur J Hum Genet 2011;19:617-623.

3 Krzystolik K, Stopa M, Kuprjanowicz L, Drobek-Slowik M, Cybulski C, Jakubowska A, Gronwald J, Lubiński J, Lubiński W: Pars plana vitrectomy in advanced cases of Von Hippel-Lindau eye disease. Retina 2016;36:325-334.

-4 McDonald HR, Schatz H, Johnson RN, Abrams GW, Brown GC, Brucker AJ, Han DP, Lewis H, Mieler WF, Meyers S: Vitrectomy in eyes with peripheral retinal angioma associated with traction macular detachment. Ophthalmology 1996;103:329-335.

5 Gaudric A, Krivosic V, Duguid G, Massin P, Giraud S, Richard S: Vitreoretinal surgery for severe retinal capillary hemangiomas in von Hippel-Lindau disease. Ophthalmology 2011;118:142-149.

-6 Majji AB: Paramacular Von Hippel angioma with tractional macular detachment. Ophthalmic Surg Lasers 2002;33:145-147.

7 Mariotti C, Giovannini A, Reibaldi M, Nicolai M, Saitta A: 25-gauge vitrectomy combined with halffluence photodynamic therapy for the treatment of juxtapapillary retinal capillary hemangioma: a case report. Case Rep Ophthalmol 2014;5:162-167.

8 Garcia-Arumí J, Sararols LH, Cavero L, Escalada F, Corcóstegui BF: Therapeutic options for capillary papillary hemangiomas. Ophthalmology 2000;107:48-54.

-9 Ridley M, Green J, Johnson G: Retinal angiomatosis: the ocular manifestations of von Hippel-Lindau disease. Can J Ophthalmol 1986;21:276-283.

10 Parmar DN, Mireskandari K, McHugh D: Transpupillary thermotherapy for retinal capillary hemangioma in von Hippel-Lindau disease. Ophthalmic Surg Lasers 2000;31:334-336.

11 Fortunato M, Di Pietro R, Gravina L, Maggi R, Ubaldi A, Vadalà P: Photodynamic therapy in von HippelLindau disease in children. J Pediatr Ophthalmol Strabismus 2009;46:376-379.

12 Dahr SS, Cusick M, Rodriguez-Coleman H, Srivastava SK, Thompson DJ, Linehan WM, Ferris FL 3rd Chew EY: Intravitreal anti-vascular endothelial growth factor therapy with pegaptanib for advanced von Hippel-Lindau disease of the retina. Retina 2007;27:150-158.

13 Peng CH, Cheng CK, Li SC, Mu SC, Lee CC: Relaxing retinectomy for tractional retinal detachment with retinal capillary hemangiomas in genotypically confirmed von Hippel-Lindau disease. Retin Cases Brief Rep 2010;4:309-311.

14 Peyman GA, Rednam KR, Mottow-Lippa L, Flood T: Treatment of large von Hippel tumors by eye wall resection. Ophthalmology 1983;90:840-847. 


\section{Case Reports in Ophthalmology}
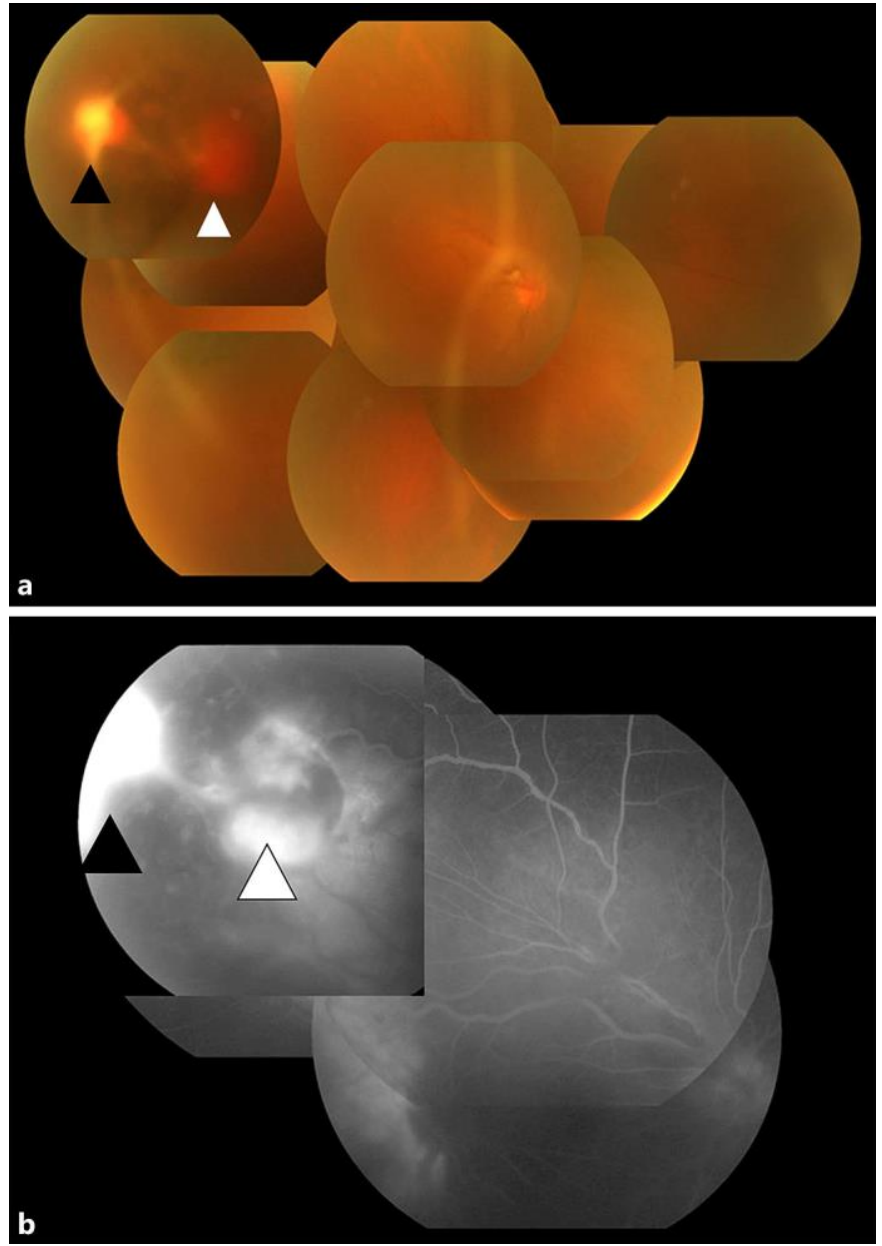

Suzuki et al: Vitrectomy for Tractional Retinal Detachment with Twin Retinal Capillary Hemangiomas in a Patient with Von Hippel-Lindau Disease: A Case Report

Fig. 1. Fundus photograph (a) and fluorescein angiography (b) in the right eye of a patient with VHL with twin retinal capillary hemangiomas. Around the temporal upper area, twin connected retinal capillary hemangiomas were observed with papillary diameter sizes of 2 and 3.5, showing peripheral fibrin deposition and exudative retinal detachment extending to the posterior pole. Fluorescein angiography revealed substantial leakage from the hemangioma on the peripheral side. $\boldsymbol{\Delta}=$ Peripheral hemangioma; $\Delta=$ posterior pole hemangioma. 


\section{Case Reports in Ophthalmology}

(C) 2016 The Author(s). Published by S. Karger AG, Basel www.karger.com/cop

Suzuki et al: Vitrectomy for Tractional Retinal Detachment with Twin Retinal Capillary Hemangiomas in a Patient with Von Hippel-Lindau Disease: A Case Report
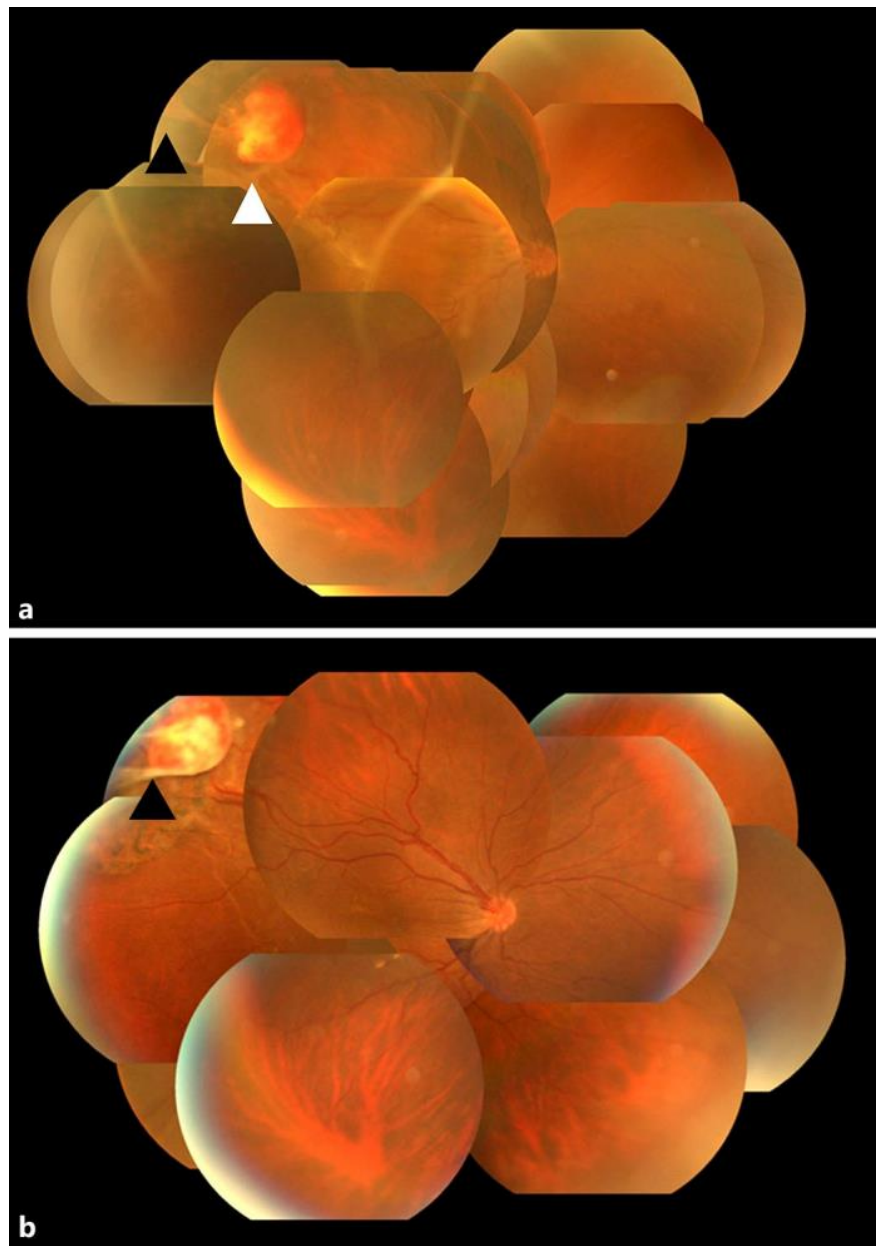

Fig. 2. Fundus photographs obtained before (a) and after (b) vitreous surgery. Tractional retinal detachment occurred following laser photocoagulation, yet the retina became reattached following the operation. $\boldsymbol{\Delta}=$ Peripheral hemangioma; $\Delta=$ posterior pole hemangioma . 


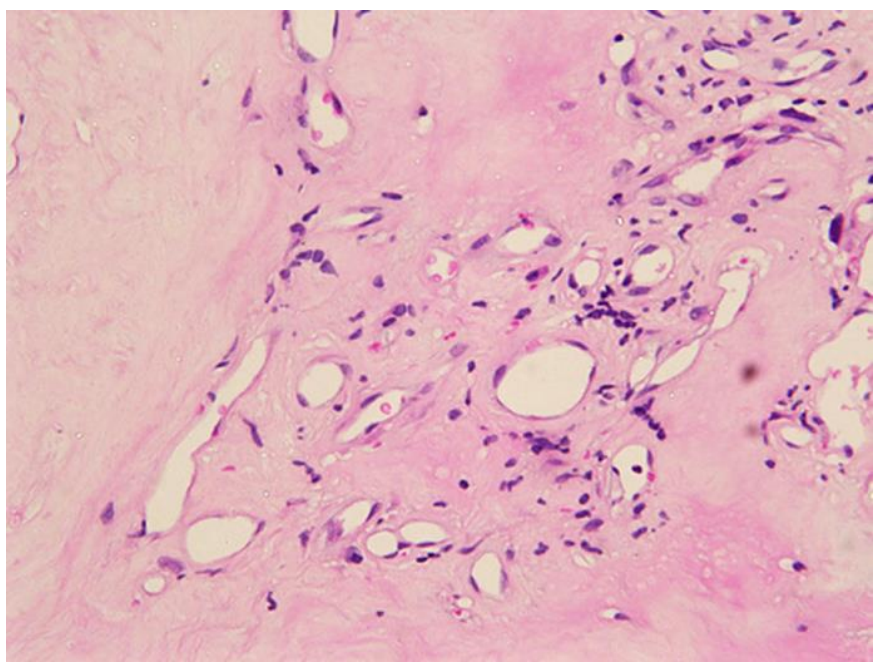

Fig. 3. Findings of the pathological examination. During a pathological examination of the tissue of the extracted hemangioma, an aggregation of blood vessels was confirmed in part of the hyalinized tissue via hematoxylin-eosin staining. 